\title{
The Subtleties of Entanglement and its Role in Quantum Information Theory
}

\author{
Rob Clifton \\ Department of Philosophy, 1001 Cathedral of Learning, \\ University of Pittsburgh, Pittsburgh, PA 15260 \\ (E-mail: rclifton@ @itt.edu)
}

March 12, 2001

\section{Introduction}

For years, since the EPR 'paradox' (1935) and Schrödinger's $(1935,1936)$ work on entanglement in the 30's, philosophers of science have struggled to understand quantum correlations. By the end of the 60's, Bell's theorem (1964) became widely recognized as establishing that these correlations cannot be explained through the operation of local common causes. Yet it was also clear that, by themselves, quantum correlations cannot be exploited to transmit information between the locations of entangled systems. Many metaphors were developed by philosophers in the 70's and 80's to characterize these apparently nonlocal yet curiously benign correlations. They were variously taken to involve 'passion-at-a-distance', 'nonrobustness', 'relational holism', and 'randomness in harmony' (see, respectively, the contributions of Shimony, Redhead, Teller and Fine to Cushing and McMullin (1989)). However, the impact of these philosophical discussions on the 
consciousness of the practicing physicist was virtually negligible. That quantum nonlocality was a fact of nature worth reckoning with was treated at about the same level of seriousness that scientists regard evidence for telepathy. In his Will to Believe, William James described the scientist's attitude towards telepathy thus:

Why do so few 'scientists' even look at the evidence for telepathy, so called? Because they think, as a leading biologist, now dead, once said to me, that even if such a thing were true, scientists ought to band together to keep it suppressed and concealed. It would undo the uniformity of Nature and all sorts of other things without which scientists cannot carry on their pursuits. But if this very man had been shown something which as a scientist he might do with telepathy, he might not only have examined the evidence, but even have found it good enough.

Since the beginning of the 90's, when the interest in quantum information theory began to explode, physicists' attitudes have changed dramatically, and James' observations about telepathy now seem downright prophetic! Witness the following passage from the introduction to Popescu and Rohrlich's (1998) 'The Joy of Entanglement':

... today, the EPR paradox is more paradoxical than ever and generations of physicists have broken their heads over it. Here we explain what makes entanglement so baffling and surprising. But we do not break our heads over it; we take a more positive approach to entanglement. After decades in which everyone talked about entanglement but no one did anything about it, physicists have begun to do things with entanglement.

What entanglement is now known to do (among other things) is increase the capacity of classical communication channels-so-called 'entanglement-assisted 
communication'. No longer is entanglement the deus ex machina philosophers' metaphors would lead us to believe. Indeed, physicists have now developed a rich theory of entanglement storage and retrieval with deep analogies to the behaviour of heat as a physical resource in classical thermodynamics.

My aim in this paper is a modest one. I do not have any particular thesis to advance about the nature of entanglement, nor can I claim novelty for any of the material I shall discuss (much of which is now readily accessible through excellent texts: Preskill (1998), Lo et al (1998), Bouwmeester et al (2000), and Nielsen and Chuang (2000)). My aim is simply to raise some questions about entanglement that spring naturally from certain developments in quantum information theory and are, I believe, worthy of serious consideration by philosophers of science. In section 2, I shall discuss different senses in which a bipartite quantum state can be said to be 'nonlocal'. All the different senses collapse into a single concept when the state is pure, but conceptually novel questions arise when the state at issue is mixed. In section 3, I will limit my discussion to the two paradigm cases of entanglement-assisted communication: dense coding and teleportation. Finally, in section 4, I shall discuss different kinds/degrees of entanglement and give a whirlwind tour of the basics of 'entanglement thermodymanics'. Space limitations force me to assume some prior acquaintence with elementary quantum mechanics, though I have endeavored to keep my discussion as self-contained and nontechnical as possible.

\section{Different Manifestations of Nonlocality}

Suppose we have two spatially separated observers, Alice and Bob, and a source that creates identically prepared pairs of particles. One member of each pair goes to Alice and the other to Bob. If Alice and Bob measure various observables on their particles, there will, in general, be correlations between the measurement results they obtain. The quantum state of each particle pair will be represented by 
a density operator $\rho$ on a tensor product $H^{A} \otimes H^{B}$ of two Hilbert spaces, with the predicted correlation between any given Alice observable $A$ and Bob observable $B$ given by $\langle A \otimes B\rangle_{\rho}=\operatorname{Tr}[\rho(A \otimes B)]$. Call a state $\rho$ Bell correlated if there are spin-type observables $A_{i}=A_{i}^{*}=A_{i}^{-1}, B_{i}=B_{i}^{*}=B_{i}^{-1}, i=1,2$, such that

$$
\left|\left\langle A_{1} \otimes B_{1}\right\rangle_{\rho}+\left\langle A_{1} \otimes B_{2}\right\rangle_{\rho}+\left\langle A_{2} \otimes B_{1}\right\rangle_{\rho}-\left\langle A_{2} \otimes B_{2}\right\rangle_{\rho}\right|>2
$$

Then Bell's theorem tells us that if $\rho$ is Bell correlated, its correlations cannot be simulated in any local hidden variables (LHV) model. Being Bell-correlated is, therefore, one sense in which a state-understood simply as a catalogue of correlations_can be said to be 'nonlocal'.

I am well aware that some quantum physicists remain committed to the view that the absence of an LHV model for correlations does not entail that they are nonlocal, but simply 'nonclassical' (e.g., Fuchs and Peres 2000). Their escape typically involves arguing that assuming the existence of hidden variables presupposes a form of realism inappropriate in quantum theory. On the other hand, other physicists have recently taken to calculating the average number of classical bits that would need to be 'communicated between the particles' to simulate their Bell correlations (Steiner 1999, Brassard et al 1999, Massar et al 2000). This has apparently been done in ignorance of similar earlier work by Maudlin (1994), and it would be interesting to determine how all these results are related. But the philosophical payoff seems clear: If we can show in purely information-theoretic terms that no local account of Bell correlations is possible, wouldn't that show that realism is a red-herring? Or is the use of classical information theory as a benchmark inappropriate in the quantum context? Since in 'quantum information' theory, it is typically the medium and not the method of calculating the information content of the message that is 'quantum', I shall proceed, tentatively, on the assumption that the answer to the first question, but not the second, is 'Yes'.

A state $\rho$ is called a product state just in case there are density operators $\rho^{A}$ and $\rho^{B}$ on $H^{A}$ and $H^{B}$, respectively, such that $\rho=\rho^{A} \otimes \rho^{B}$. More generally, a 
state $\rho$ is called separable just in case it can be written as a convex combination of product states

$$
\rho=\sum_{i} p_{i} \rho_{i}^{A} \otimes \rho_{i}^{B}, \quad p_{i} \in[0,1], \quad \sum_{i} p_{i}=1
$$

Clearly the correlations of separable states can be simulated locally, because we may think of the separate quantum states $\rho_{i}^{A}$ and $\rho_{i}^{B}$ as themselves supplying the required local hidden variables, with the probabilities $p_{i}$ representing our ignorance about which hidden variables obtain in a given measurement trial. Thus, a state is separable if and only if its correlations admit a quantum LHV model.

So Bell correlated states are nonlocal while separable states are local. What about nonseparable, or entangled, states: Are they always nonlocal? It certainly does not automatically follow from the fact that a state admits no quantum LHV model that it does not admit any LHV model whatsoever. However, if an entangled state is pure, it can be shown that it must be Bell correlated (Popescu and Rohrlich 1992), and so the answer is 'Yes' for pure entangled states. Not so in the mixed case. Consider the following 'Werner state' in $2 \times 2$ dimensions (Werner 1989):

$$
\rho_{W}=\left(1-\frac{1}{\sqrt{2}}\right) \frac{1}{4} I \otimes I+\frac{1}{\sqrt{2}}\left|\Psi_{-}\right\rangle\left\langle\Psi_{-}\right|,
$$

where $\left|\Psi_{-}\right\rangle$is the infamous EPR-Bohm singlet state of two spin-1/2 particles. Since spin operators are traceless, the maximum possible Bell correlation in state $\rho_{W}$ is determined by $\frac{1}{\sqrt{2}}$ times the maximum Bell correlation achievable in the singlet state, which is $2 \sqrt{2}$. Thus $\rho_{W}$ is not Bell correlated. Nevertheless, $\rho_{W}$ is entangled - not because the particular convex combination used above to define $\rho_{W}$ involves the singlet state (which is, of course, not a product state), but because there is no other way to rewrite $\rho_{W}$ as a convex combination of product states. Nevertheless, while $\rho_{W}$ does not admit a quantum LHV model, Werner (1989) was able to construct a LHV model for its correlations.

It would be natural to conclude that since an entangled state need not be Bell correlated—and can even admit a LHV model—some entangled states are surely 
local. One might object to this by observing that every convex decomposition of a mixed entangled state into pure states must involve at least one entangled pure state (else the mixed state would be separable); and, therefore, since pure entangled states are nonlocal, mixed entangled states should be thought of as nonlocal too. But this reasoning runs afoul of well-known difficulties with the ignorance interpretation of mixtures. A mixed density operator like $\rho_{W}$ need not result from physically mixing, in known proportions, pure states of two spin-1/2 particles. It could result, instead, from reducing the density matrix of a larger system in a pure entangled state with the spin-1/2 particles-a state in which there is no fact of the matter about what pure state the particles occupy for anyone to be ignorant about! Moreover, even if we knew that a mixed state has been produced by physically mixing pure states, there are many inequivalent ways to mix and produce the same density matrix. For example, the 'maximally mixed' product state $(I \otimes I) / 4$ of two spin-1/2 particles is surely local, and can be produced by an equal mix of the four product pure states

$$
\left|\downarrow_{z}\right\rangle \otimes\left|\downarrow_{z}\right\rangle,\left|\downarrow_{z}\right\rangle \otimes\left|\uparrow_{z}\right\rangle,\left|\uparrow_{z}\right\rangle \otimes\left|\downarrow_{z}\right\rangle,\left|\uparrow_{z}\right\rangle \otimes\left|\uparrow_{z}\right\rangle .
$$

Yet $(I \otimes I) / 4$ can also be produced by physically mixing, in equal proportions, the following four entangled eigenstates of the 'Bell operator':

$$
\begin{aligned}
& \left|\Psi^{ \pm}\right\rangle=\frac{1}{\sqrt{2}}\left(\left|\downarrow_{z}\right\rangle \otimes\left|\uparrow_{z}\right\rangle \pm\left|\uparrow_{z}\right\rangle \otimes\left|\downarrow_{z}\right\rangle\right), \\
& \left|\Phi^{ \pm}\right\rangle=\frac{1}{\sqrt{2}}\left(\left|\downarrow_{z}\right\rangle \otimes\left|\downarrow_{z}\right\rangle \pm\left|\uparrow_{z}\right\rangle \otimes\left|\uparrow_{z}\right\rangle\right) .
\end{aligned}
$$

In the absence of further knowledge of the mixing process, the possibility of producing $(I \otimes I) / 4$ by mixing eigenstates of the Bell operator shows only that there is a nonlocal quantum hidden variables model of the state $(I \otimes I) / 4$, not that there can be no local one! So we see that it is in general fallacious to take the locality properties of the pure components of a mixture to be indicative of the locality or nonlocality of the mixture itself. 
There is, however, a more interesting objection to the claim that certain entangled states, like the Werner state, are local. What if we require more of a LHV model than that it simply reproduce the correlations between Alice's and Bob's measurement outcomes? Suppose prior to making the measurements on their particles from which they calculate their correlations, we allow Alice and Bob to 'preprocess' the particles by performing arbitrary local operations on them and communicating about the results they obtain. By this is meant the following.

A nonselective local operation on Alice's particle is any 'completely positive, linear, and trace-preserving' transformation of its density operator that leaves unchanged the state of Bob's particle. Such a transformation need not be unitary. For example, it could be brought about by Alice first combining her particle with an ancilla system which has no prior entanglement with either Alice's or Bob's particle, then executing a unitary transformation on the combined particle+ancilla system, and, finally, tracing out the ancilla to get a reduced state for Alice's particle again. Alice could also perform local measurements on her particle (whose outcome statistics will, in general, only be representable by positive operators rather than projections), and communicate to Bob her results so that he can coordinate the local operations he performs on his particle with the outcomes Alice gets from hers. In particular, we can allow Alice and Bob to perform non-tracepreserving selective local operations, in which they drop from further consideration certain members of their initial ensemble - on the basis of certain measurements results they obtain - and communicate classically between them to ensure agreement about which particle pairs are dropped.

The remarkable thing is that it is possible, after Alice and Bob avail themselves of local operations and classical communication on an initially 'local' but entangled particle pair, for the final state of the surviving ensemble of particle pairs to be Bell correlated! (See Popescu (1995) and Mermin (1996).) As it happens, this only works for higher-dimensional Werner states, not the simple $2 \times 2$ state 
$\rho_{W}$ we have considered. In order to 'display' $\rho_{W}$ 's nonlocality, we need to allow Alice, and similarly Bob, the additional freedom to perform collective operations on all of her particles at once (Peres 1996). These operations will still be local, insofar as they do not affect the states of Bob's particles (even if they might introduce entanglement amongst Alice's). Conventional wisdom then dictates that, after all is said (between Alice and Bob) and done (by their collective local operations), an initial entangled state that, as a result of 'preprocessing' changes to a Bell correlated one, should be understood as nonlocal. For example, Gisin (p. 271) writes 'A state that is explicitly nonlocal after some local operations does not deserve the qualification of local... because reproducing all correlations [of the initial state] is not enough'. Similarly, Popescu and Rohrlich $(1998,41)$ state: 'Alice and Bob could never obtain nonlocal states from local states by using only local interactions'.

What are we to make of this conventional wisdom? It is certainly true that if we demand there exist an extended LHV model, not just for the correlations of the initial state, but for all of Alice and Bob's preprocessing and the Bell correlations of their final state, then the model cannot be local. Moreover, it appears we are not putting a LHV model at a disadvantage by requiring it to model all of this; for in confining Alice and Bob to the use of local operations on their particles and normal causal communication, we are not adding any 'extra nonlocality' between the particles for the model to explain. Still, what precisely is the argument for taking the original unprocessed entangled state to intrinsically possess nonlocal (as opposed to merely entangled) correlations?

The only way I can see to justify such a claim is if the following conjecture could be proved: If an HV model $M$ of the correlations of an initial entangled state has an extension to- - or is the restriction of - a more encompassing HV model of all the collective local operations and classical communication needed to produce a Bell correlated state, then the model $M$ of the initial state must already have 


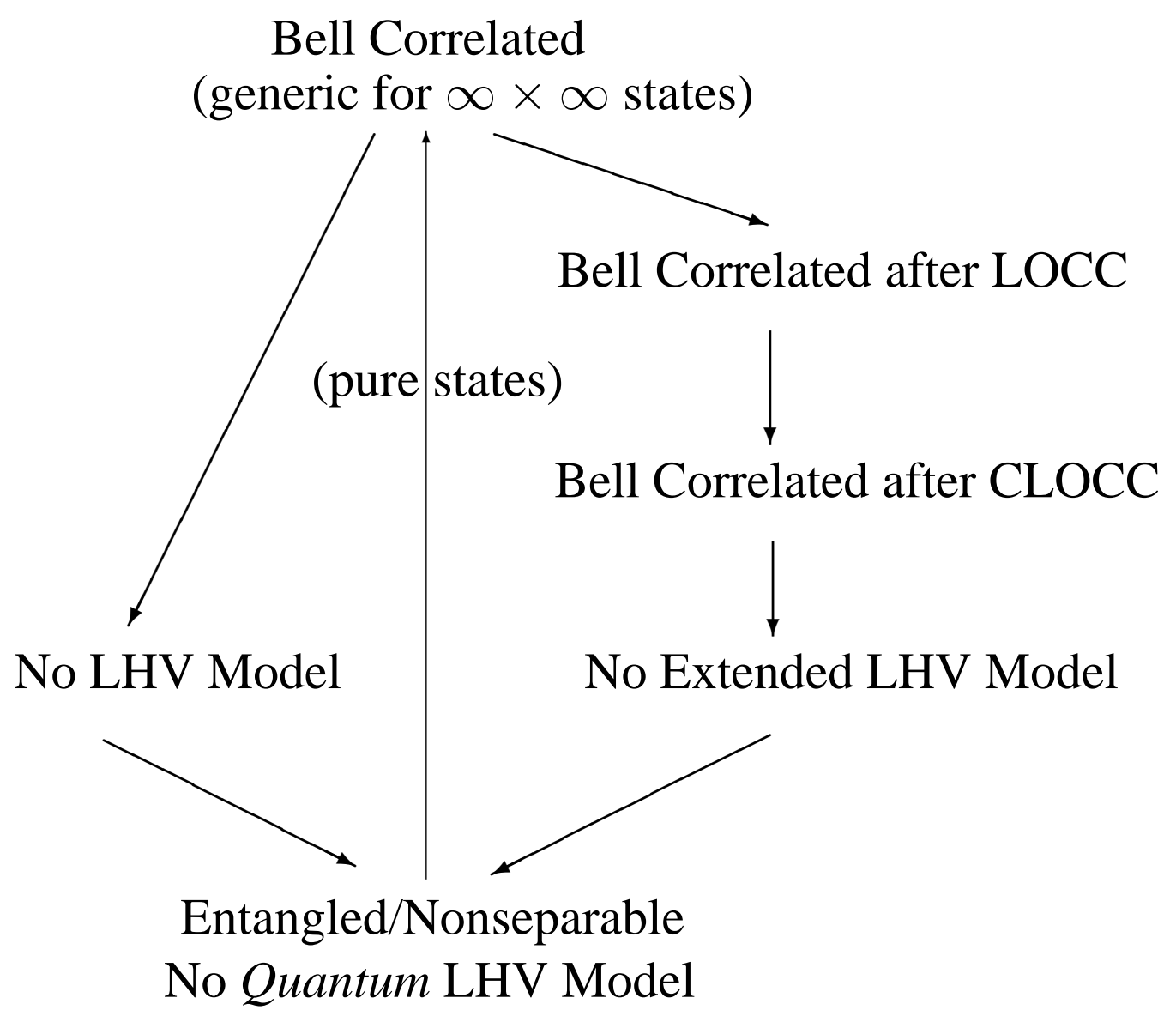

Figure 1: Different Manifestations of Nonlocality (LHV = Local Hidden Variables, $\mathrm{LO}=$ Local Operations, $\mathrm{CC}=$ Classical Communication, $\mathrm{LOCC}=\mathrm{LO}+$ $\mathrm{CC}, \mathrm{CLOCC}=$ Collective $\mathrm{LOCC}$ )

contained nonlocal elements. If true, this would entail that Werner's LHV model for $\rho_{W}$ 's correlations does not extend to any adequate model-local or not-of the production of the final state after (collective) preprocessing. Similarly, the truth of my conjecture would mean that Bohm's theory, which is certainly capable of reproducing all the predictions of quantum theory associated with Alice and Bob's preprocessing, yields a nonlocal dynamics between two particles in the Werner state (understood as the reduced state of a larger three particle pure state, if necessary), notwithstanding its lack of Bell correlation. However, even if my conjecture is true, I am unaware of any general argument that every entangled 
state must yield to local operations and classical communication and eventually produce a Bell correlated state. Morever, we shall see in Section 4 that certain entangled states definitely resist preprocessing into singlet states.

Figure 1 above summarizes the different senses of nonlocality I have discussed, and their logical relations. Note that for pure states, all distinctions in the diagram collapse. It can also be shown that the Bell correlated states of infinite-dimensional systems are generic (Clifton et al 1999), and therefore, for all practical purposes, the diagram collapses in that case as well.

\section{Entanglement-Assisted Communication}

I turn now to discuss two novel and conceptually puzzling practical uses of entanglement: dense coding (Bennett and Wiesner 1992) and teleportation (Bennett et al 1993). Both involve forms of communication between Alice and Bob in which it looks like Bob is able to receive more information from Alice than she actually sent him.

In dense coding (see Figure 2 below), the aim is for Alice to convey to Bob two classical bits of information, or 'cbits' for short. They initially share a pair of spin-1/2 particles, or 'qubits', in the singlet state $\left|\Psi^{-}\right\rangle$. While Bob holds on to his particle, Alice has the option of doing nothing to hers, or performing one of three unitary operations given by the Pauli spin operators $\sigma_{x}, \sigma_{y}$, or $\sigma_{z}$. After exercising her option, the final joint state of the particles will either be $\left|\Psi^{-}\right\rangle,\left(\sigma_{x} \otimes I\right)\left|\Psi^{-}\right\rangle$, $\left(\sigma_{y} \otimes I\right)\left|\Psi^{-}\right\rangle$, or $\left(\sigma_{z} \otimes I\right)\left|\Psi^{-}\right\rangle$. These four states are easily seen to be mutually orthogonal (use $\sigma_{x} \sigma_{y}=i \sigma_{z}+$ cyclic permutations, and $\left\langle\Psi^{-}\left|\left(\sigma_{x, y, z} \otimes I\right)\right| \Psi^{-}\right\rangle=0$ ) and are, in fact, the four Bell operator eigenstates (up to phase). Thus, Alice can now send her qubit to Bob, who can combine it with his own qubit and measure the Bell operator on them jointly to perfectly distinguish which of the four options Alice took.

What is 'dense' about this coding is that it apparently exceeds a well-known 


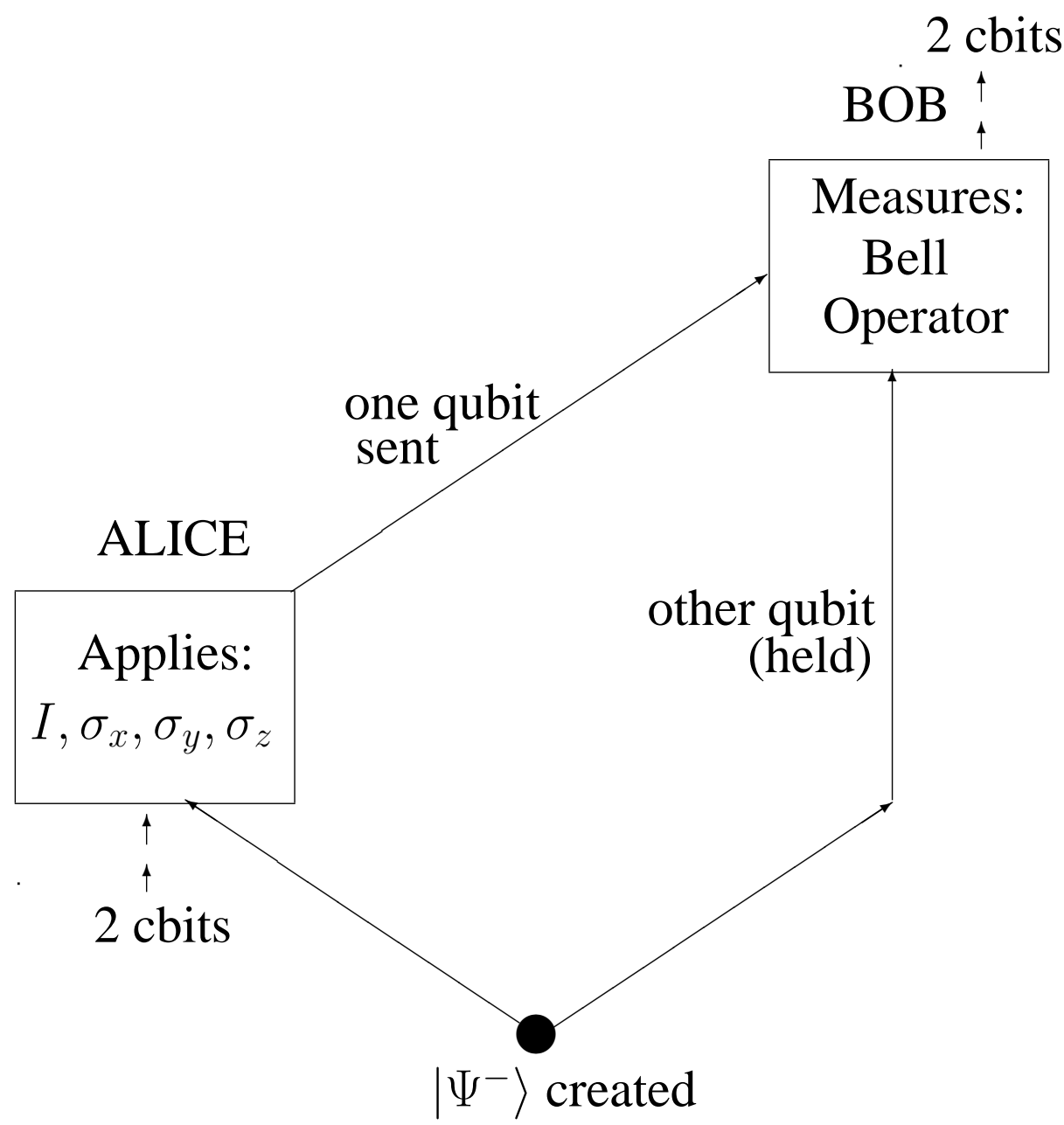

Figure 2: Dense Coding

limit—called the Holevo bound-on the capacity of qubits to carry classical information. For a consequence of this bound is that a state of $n$ qubits can be used between a sender and receiver to transmit at most $n$ cbits (Nielson and Chuang 2000, Exercise 12.3). Yet Bob receives two cbits with only one qubit changing hands! On closer inspection, however, there is no contradiction. The setup presupposed for Holevo's bound says nothing about which qubits must 'change hands' but, rather, assumes only that the sender encodes her information by performing operations on a collection of $n$ qubits, and the receiver retrieves as much 
of that information as possible by making measurements on that same collection. Nothing precludes the sender from operating locally only on some subset of the $n$ 'information carrying qubits' which, if that subset is entangled with the rest, can still produce a nontrivial change in their total state. Thus, from the point of view of Holevo's bound, what Alice actually does in the dense coding is to code her two cbits into the state of two qubits, and Bob chooses a Bell operator measurement that allows him to retrieve as much information as he possibly can. Note, also, that Alice's trick wouldn't work if her qubit weren't entangled with Bob's. For example, replacing $\left|\Psi^{-}\right\rangle$by $\left|\uparrow_{z}\right\rangle \otimes\left|\downarrow_{z}\right\rangle$ would only leave Bob, at the end of the protocol, with one of the two orthogonal states $\left|\uparrow_{z}\right\rangle \otimes\left|\downarrow_{z}\right\rangle,\left|\downarrow_{z}\right\rangle \otimes\left|\downarrow_{z}\right\rangle$ (up to phase), from which he can distinguish only 1 cbit about Alice's choiceconsistent with the Holevo bound on the information transmittable using only a single qubit.

Though there is no outright contradiction with the Holevo bound in the entangled case, it is hard to escape the sense that the qubit in Bob's possession could not possibly carry any of the information he finally receives. After all, Bob has his qubit in his hands well in advance of Alice deciding what information she wants to send by choosing between the four unitary operations. The Holevo bound is cleverly silent about this, leaving us the option of filling in how the $n$ cbits the sender encodes get 'distributed amongst' the $n$ encoding qubits. Moreover, while the bound allows us to say that if Bob looks at either qubit in isolation he can get up to 1 cbit of information, it is easy to see that, in fact, absolutely no information about Alice's unitary operation can be obtained in that way. Should we, then, resist the temptation to fill in the story and marvel in the fact that quantum holism gives merely the appearance of nonlocal information transfer between the qubits without actually instantiating it?

Consider the following classical analogue of dense-coding (suggested to me by Arthur Cunningham). A source produces a pair of cards. One card, which is 
blank, goes to Alice; the other, on which is (randomly) written one of four ordered pairs $\left(x_{2}, y_{2}\right)=(0,0),(0,1),(10)$, or $(1,1)$, goes to Bob. Alice chooses one of these same four ordered pairs, call it $\left(x_{1}, y_{1}\right)$, to write on her card, and then sends it to Bob. Finally, Bob combines the information from the two cards and computes the binary sum $\left(x_{1} \oplus x_{2}, y_{1} \oplus y_{2}\right)=(0,0),(0,1),(10)$, or $(1,1)$. In this way, Alice can communicate to Bob one of the latter four pairs, i.e., 2 cbits, by writing on her card the appropriate pair $\left(x_{1}, y_{1}\right)$. Note that by looking at either card on its own, Bob can infer nothing about the 2 cbits Alice is trying to send him-no (relevant) information is carried by either card in isolation. Thus, even in the absence of quantum holism, there is no reason to think that the information carried by a communication channel must be parcelled out amongst the component physical systems making up the channel. It seems that dense coding does not mandate a holistic metaphysics after all.

Unfortunately, this classical communication protocol is disanalogous to dense coding in one important respect. In order for Alice to choose the appropriate pair $\left(x_{1}, y_{1}\right)$ with which to convey her 2 cbits, she must know in advance what is written on Bob's card. What would be the mechanism by which she could get this sort of knowledge in the quantum case? For example, Alice can learn nothing from the prior EPR correlation between the qubits, because she does not perform any measurements on her qubit! One is, therefore, tempted to conjecture that any classical simulation of dense coding, if it does not involve holistic elements, must still involve nonlocal effects of one sort or another.

In teleportation (see Figure 3 below), Alice and Bob again share a pair of spin$1 / 2$ particles in the singlet state $\left|\Psi^{-}\right\rangle$, but now they swap their equipment: Alice first measures the Bell operator, and then Bob applies one of the four unitary transformations $I, \sigma_{x}, \sigma_{y}$, or $\sigma_{z}$. The aim is for Alice to transmit to Bob the state $|\phi\rangle$ of another 'ancilla' qubit. She does so by exploiting the correlations in their 'channel state' $\left|\Psi^{-}\right\rangle$and sending Bob 2 cbits rather than a qubit. The initial 
ancilla+channel state is $|\phi\rangle \otimes\left|\Psi^{-}\right\rangle$. One can verify that the unitary operator which permutes the state of the ancilla with Bob's qubit can be written as

$$
P=1 / 2\left(I \otimes I \otimes I+\sigma_{x} \otimes I \otimes \sigma_{x}+\sigma_{y} \otimes I \otimes \sigma_{y}+\sigma_{z} \otimes I \otimes \sigma_{z}\right) .
$$

Therefore,

$$
\begin{aligned}
|\phi\rangle \otimes\left|\Psi^{-}\right\rangle= & -P\left(\left|\Psi^{-}\right\rangle \otimes|\phi\rangle\right)\left(\text { using the anti-symmetry of }\left|\Psi^{-}\right\rangle\right) \\
= & -1 / 2\left[\left|\Psi^{-}\right\rangle \otimes|\phi\rangle+\left(\sigma_{x} \otimes I\right)\left|\Psi^{-}\right\rangle \otimes \sigma_{x}|\phi\rangle\right. \\
& \left.+\left(\sigma_{y} \otimes I\right)\left|\Psi^{-}\right\rangle \otimes \sigma_{y}|\phi\rangle+\left(\sigma_{z} \otimes I\right)\left|\Psi^{-}\right\rangle \otimes \sigma_{z}|\phi\rangle\right]
\end{aligned}
$$

The protocol can now be read directly off this last expression. Alice first measures the Bell operator, collapsing the joint state of the ancilla qubit and hers into one of the four Bell eigenstates, say $\left(\sigma_{y} \otimes I\right)\left|\Psi^{-}\right\rangle$. As a result, Bob's qubit is left in the correlated state $\sigma_{y}|\phi\rangle$. Alice then communicates to Bob which of the four measurement outcomes she got, and Bob applies the corresponding unitary operation, in this case $\sigma_{y}$, to his qubit. After doing so, he obtains $\sigma_{y}^{2}|\phi\rangle=|\phi\rangle$ and, thereby, creates an exact replica of the ancilla qubit's state at his location.

In a sense, teleportation is a form of 'dense coding', because, while a normal classical communication channel would need to carry an infinite (or, at least, arbitrarily large) amount of information to convey to Bob the expansion coefficients of the state $|\phi\rangle$, Alice manages to get by with sending him only 2 cbits. This again raises the question of whether a convincing story can be told here about the 'flow of information' (nonlocally?!, backwards in time?!) between Alice and Bob (see Duwell 2000). But note that there is an important difference from dense coding: if there is information transfer in teleportation at all, it occurs entirely at the quantum level. A successful run of the protocol does not require that Alice know in advance the state $|\phi\rangle$ she is teleporting; nor can it end in Bob's knowing the identity of this state, because (as is well-known) there is no way for Bob to determine the quantum state of a single system! Perhaps some new concept of hidden quantum information transfer is called for here? (See Cerf and Adami 1997, Deutsch 


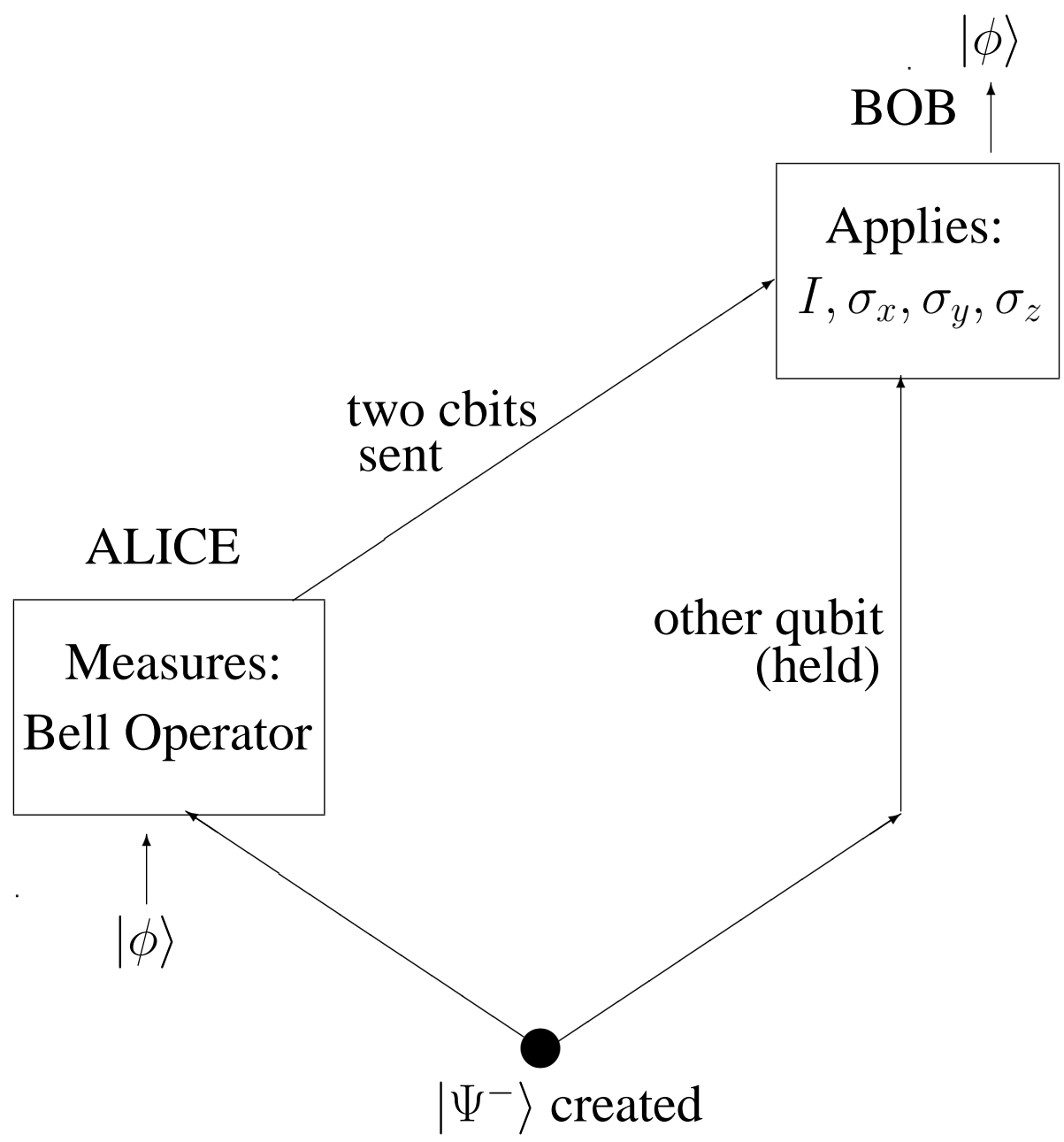

Figure 3: Teleportation

and Hayden 1999.)

We can also consider imperfect teleportation, where the state Bob ends up creating at the end of the protocol is not exactly the ancilla's state, but something close. It turns out that imperfect teleportation with a 'fidelity' higher than any classical analogue can achieve is always possible when the singlet channel state Alice and Bob share is replaced by any Bell correlated entangled state (Horodecki et al 1996). However, high fidelity teleportation is also possible using the Werner state, which is not Bell correlated (Popescu 1994). One can either take this to be a 
sign that the Werner state is, after all, nonlocal, or see it as showing that explaining the success of teleportation does not require nonlocality.

This issue can also be attacked more directly by taking a closer look at the correlations of the channel state that are actually involved in the teleportation protocol (Źukowski 2000). LHV models will be committed to all 'Bell teleportation inequalities' of the form

$\left|\left\langle f_{1} \otimes \sigma_{1}\right\rangle_{\left|\phi_{1}\right\rangle\left\langle\phi_{1}\right| \otimes \rho}+\left\langle f_{1} \otimes \sigma_{2}\right\rangle_{\left|\phi_{1}\right\rangle\left\langle\phi_{1}\right| \otimes \rho}+\left\langle f_{2} \otimes \sigma_{1}\right\rangle_{\left|\phi_{2}\right\rangle\left\langle\phi_{2}\right| \otimes \rho}+\left\langle f_{2} \otimes \sigma_{2}\right\rangle_{\left|\phi_{2}\right\rangle\left\langle\phi_{2}\right| \otimes \rho}\right| \leq 2$,

where $f_{1,2}$ are bivalent functions of Alice's Bell operator, $\left|\phi_{1,2}\right\rangle$ are alternatives for the teleported state, $\rho$ is the 'quantum channel' state, and $\sigma_{1,2}$ are spin components of Bob's particle. It can be shown that if the teleportation fidelity exceeds a certain threshold, at least one such inequality must be violated, but that no violations occur in the Werner state (Clifton and Pope 2000). While the verdict is still out, this suggests that the ability of a quantum state to facilitate nonclassical teleportation should not be taken as a litmus test for the nonlocality of the state.

\section{Entanglement Thermodynamics}

There is one further trick that can be performed with teleportation that would appear particularly difficult to classically simulate: entanglement swapping. Suppose the ancilla qubit whose state Alice teleports does not actually have a definite state of its own, but is entangled via the singlet state $\left|\Psi^{-}\right\rangle$with yet another qubit. Let's label that qubit 0 , the ancilla qubit 1, and Alice and Bob's channel qubits 2 and 3, respectively. Now follow through the exact same protocol as before, starting instead with the initial state $\left|\Psi^{-}(0,1)\right\rangle \otimes\left|\Psi^{-}(2,3)\right\rangle=$ $-P\left(\left|\Psi^{-}(0,3)\right\rangle \otimes\left|\Psi^{-}(1,2)\right\rangle\right)$. Clearly the result, in all cases, will be that qubits $1+2$ will be left in a Bell operator eigenstate, and qubits $0+3$ will now be entangled in the singlet state instead! It appears that there is enough 'substance' to entanglement that it can be 'moved' from one pair of particles to another. Note, 
however, that this swapping process has not led to a net increase in the entanglement between Alice and Bob; teleportation destroys the singlet state entanglement they initially shared in their channel state, as it replaces it with singlet entanglement between 0 and 3 .

Since the entanglement of the channel state in teleportation will inevitably get used up (and, similarly, Alice and Bob will use up their shared entanglement in dense coding when she passes her qubit to Bob), it is natural to develop a theory of entanglement as a physical resource for performing further 'informational work'. Entanglement thermodynamics is that theory, and yields constraints on our ability to store, retrieve and manipulate entanglement that are analogous to what classical thermodynamics tells us about heat.

The Fundamental Law of Entanglement Thermodynamics asserts: Entanglement between systems in two regions cannot be created merely by collective local operations on the systems and classical communication between the regions (i.e., CLOCC). More precisely, any reasonable measure $E$ of entanglement must satisfy

$$
\rho \stackrel{C L O C C}{\longmapsto}\left\{\rho_{i}, p_{i}\right\} \Longrightarrow E(\rho) \geq \sum_{i} p_{i} E\left(\rho_{i}\right)
$$

where the antecedent of this conditional signifies that Alice and Bob have preprocessed the particles and (possibly) sorted them into subensembles $\rho_{i}$ with probabilities $p_{i}$, and the consequent says that the average entanglement left at the end of the process cannot exceed the initial entanglement that went in. Note that there is a clear analogy here with the Second Law of Classical Thermodynamics: There is no process the sole effect of which is to extract heat from the colder of two reservoirs and deliver it to the hotter reservoir. (Plenio and Vedral 1998.) Just as we cannot create refrigeration for free, i.e., without any energy or work input, we cannot produce entanglement for free, i.e., without entangling interactions between the particles.

Motivated by the problems of entanglement storage and retrieval, there are two natural measures of entanglement obeying the fundamental law. Suppose 
Alice and Bob start by sharing a large collection of pairs of particles in a state $\rho^{\otimes n}=\rho \otimes \ldots \otimes \rho(n$ times $)$. Alice collectively operates on her members of each pair, and Bob on his, with the aim of extracting $k \leq n$ singlet states between them. The entanglement of distillation of $\rho$ is the maximum fraction of singlets they can extract from $\rho^{\otimes n}$ in the asymptotic limit as $n \rightarrow \infty$ :

$$
\rho^{\otimes n} \stackrel{C L O C C}{\longmapsto}\left|\Psi^{-}\right\rangle^{\otimes k}, \quad D(\rho)=\lim _{n \rightarrow \infty} \frac{k_{\max }}{n} .
$$

(In fact, the CLOCC transformation from $\rho^{\otimes n}$ to $\left|\Psi^{-}\right\rangle^{\otimes k}$ is only required to be perfect in the asymptotic limit.) Similarly, the entanglement of formation of $\rho$ is the minimum fraction of singlets Alice and Bob need to invest to create $n$ copies of $\rho$ in the asymptotic limit:

$$
\left|\Psi^{-}\right\rangle^{\otimes k^{\prime}} \stackrel{C L O C C}{\longmapsto} \rho^{\otimes n}, \quad F(\rho)=\lim _{n \rightarrow \infty} \frac{k_{m i n}^{\prime}}{n} .
$$

By the fundamental law, it must be the case that $D(\rho) \leq F(\rho)$.

Consider, now, the special case where $\rho$ is a pure entangled state $|\Phi\rangle$. There is a scheme, due originally to Bennett et al. (1996), that shows how Alice and Bob can asymptotically distill $|\Phi\rangle^{\otimes n} \stackrel{C L O C C}{\longmapsto}\left|\Psi^{-}\right\rangle^{\otimes k}$ with an efficiency $S\left(\rho_{\Phi}\right)$, where $\rho_{\Phi}$ is the reduced density operator for either particle in the state $|\Phi\rangle$, and

$$
S\left(\rho_{\Phi}\right)=-\operatorname{Tr}\left(\rho_{\Phi} \log _{2} \rho_{\Phi}\right)
$$

is the standard von Neumann entropy of $\rho_{\Phi}$. (Note: $S\left(\rho_{\Psi^{-}}\right)=1$, as we expect.) This scheme is reversible, in the sense that there is a scheme that asymptotically forms $\left|\Psi^{-}\right\rangle^{\otimes k^{\prime}} \stackrel{C L O C C}{\longmapsto}|\Phi\rangle^{\otimes n}$ with the same efficiency. (Details of these schemes may be found in Nielsen and Chuang (2000), Section 12.5.2.) By the Fundamental Law, no schemes can perform better. The argument for this works in complete analogy with the classical argument for the ideal efficiency of the Carnot cycle (Popescu and Rohrlich 1997). For example, if distillation could be more efficient than the Bennett et al scheme (a similar argument works for formation), then Alice and Bob would be able to start with $n$ copies of $|\Phi\rangle$ and CLOCC transform them 
into $k>n S\left(\rho_{\Phi}\right)$ singlets. They could then use the reverse of the Bennett et al scheme to CLOCC transform their $k$ singlets back into $k / S\left(\rho_{\Phi}\right)>n$ copies of $|\Phi\rangle$, in violation of the Fundamental Law. It follows that $D(\Phi)=F(\Phi)=S\left(\rho_{\Phi}\right)$.

When $\rho$ is a mixed entangled state, we can define its bound entanglement by

$$
B(\rho) \equiv F(\rho)-D(\rho)(\geq 0)
$$

Comparing this with the classical Gibbs-Helmholtz equation,

$$
T S=U-A,
$$

where $U$ is the internal energy, and $A$ the free energy, we are tempted to think of $F(\rho)$ as the 'internal entanglement' of $\rho$ and $D(\rho)$ as its 'free entanglement'. But what are the analogues of temperature $T$ and entropy $S$ ? If we took the 'entanglement entropy' of $\rho$ to be $S(\rho)$, then since the von Neumann entropy of a pure state $|\Psi\rangle$ is zero, it would follow that $F(\Psi)=D(\Psi)$ - something we already know to be true! This suggests that we further define entanglement temperature, for mixed states (when $S(\rho)>0$ ), by the formula $T(\rho) \equiv \frac{B(\rho)}{S(\rho)}$. Unfortunately, this definition turns out to have counterintuitive consequences (Horodecki et al 1998a). So the question remains: Just how deep and convincing can this thermal analogy be made?

As for the values of entanglement of distillation and formation for mixed states, remarkably little is known in general, but what is known is fascinating. Though all $2 \times 2$ entangled states $\rho$ are distillable, i.e., $D(\rho)>0$ (Horodecki et al 1997), there are higher-dimensional states, so-called bound entangled states, for which $D(\rho)=0$ (Horodecki et al 1998b). However, this does not of itself imply that $B(\rho)>0$ - and, therefore, that a genuinely irreversible entanglement manipulation process exists-because there is no general argument that one cannot get by with investing less and less entanglement in the asymptotic limit for the formation of a mixed entangled state. The first genuinely irreversible entanglement manipulation process was not found until February of this year (Vidal and Cirac 
2001), after an initially false start (Horodecki et al 2000a). Still more curious is recently found evidence for the possibility of a pair of mixed entangled states $\rho_{1,2}$ such that that $B\left(\rho_{1,2}\right)=F\left(\rho_{1,2}\right)>0$ yet $D\left(\rho_{1} \otimes \rho_{2}\right)>0$ (Shor et al 2000). If correct, this would show that bound entangled states can 'catalyze' eachother to produce free entanglement!

There is much here for philosophers of science to chew on. Clearly the thermal analogy functions as a useful heuristic for understanding entanglement and harnessing its use. But could there be more to the analogy than that? Since classical thermodynamics is a principle theory, is it appropriate to ask for a constructive underpinning, i.e., micro-theory, for 'entanglodynamics'? Could this motivate a return to the well-trodden path of hidden-variable reconstructions of quantum theory? Or does quantum theory itself supply the micro-theory via the theory of local operations? (But if it does, how should we regard the disanalogy that entanglement is still treated as a primitive, unlike its analogue heat, which is reduced to kinetic energy in statistical mechanics?) Finally, and perhaps the biggest philosophical carrot of all, could the thermal analogy be turned into a full-fledged interpretation' of quantum theory as a complete theory? As the Horodecki's (2000b) have put it:

It is characteristic that despite the dynamical development of the interdisciplinary domain of quantum information theory, there is no, to our knowledge, impact of the latter on interpretational problems. [...] Does quantum information phenomena provide objective [grounds] for the existence of a "natural" ontology inherent in the quantum formalism? 


\section{References}

Bell, J. S. (1964), 'On the Einstein-Podolsky-Rosen Paradox', Physics 1, 195200.

Bennett, C. and Wiesner, S. (1992), 'Communication via One- and Two-Particle Operators on Einstein-Podolsky-Rosen States', Phys. Rev. Lett. 69, 2881-2884.

Bennett, C., Brassard, G., Crepeau, C., Josza, R., Peres, A., and Wooters, W. (1993), 'Teleporting an Unknown Quantum State via Dual Classical and EinsteinPodolsky-Rosen Channels', Phys. Rev. Lett. 70, 1895-1899.

Bennett, C., Bernstein, H., Popescu, S., and Schumacher, B. (1996), 'Concentrating Partial Entanglement by Local Operations', Phys. Rev. A 53, 2046-2052.

Brassard, G., Cleve, R., and Tapp, A. (1999), 'The Cost of Exactly Simulating Quantum Entanglement with Classical Communication', Phys. Rev. Lett. 83, 1874-1877.

Bouwmeester, D., Ekert, A., and Zeilinger, A. (eds.) (2000), The Physics of Quantum Information, Springer-Verlag, Berlin.

Cerf, N. and Adami, C. (1997), 'Negative Entropy and Information in Quantum Mechanics', Physical Review Letters 79, 5194-5197.

Clifton, R. and Pope, D. (2000), 'On the Nonlocality of the Quantum Channel in the Standard Teleportation Protocol', forthcoming.

Clifton, R., Halvorson, H., and Kent, A. (1999), 'Non-local Correlations are Generic in Infinite-Dimensional Bipartite Systems,' Physical Review A, 61, 042101.

Cushing, J. T. and McMullin, E. (eds) (1989), Philosophical Consequences of Bell's Theorem, Notre Dame University Press.

Deutsch, D. and Hayden, P. (1999), 'Information Flow in Entangled Quantum Systems', quant-ph/9906007. 
Duwell, A. (2000), 'Explaining Information Transfer in Quantum Teleportation', in PSA 2000, Vol. 1.

Einstein, A., Podolsky. B., and Rosen, N. (1935), 'Can Quantum-Mechanical Description of Physical Reality be Considered Complete?', Phys. Rev. 47, 777780 .

Fuchs, C. A. and Peres, A. (2000), 'Quantum Theory Needs No 'Interpretation”, Physics Today 53, 70-71.

Gisin, N. (2000), 'Local Filtering', in Bouwmeester et al (2000), pp. 269-271.

Horodecki, R., Horodecki, M., and Horodecki, P. (1996), 'Teleportation, Bell's Inequalities and Inseparability', Phys. Lett. A 222, 21-25.

Horodecki, M., Horodecki, P., and Horodecki, R. (1997), 'Inseparable Two Spin1/2 Density Matrics Can Be Distilled to a Singlet Form', Phys. Rev. Lett. 78, 574-577.

Horodecki, P., Horodecki, R., and Horodecki, M. (1998a), 'Entanglement and Thermodynamical Analogies', quant-ph/9805072.

Horodecki, M., Horodecki, P., and Horodecki, R. (1998b), 'Mixed-State Entanglement and Distillation: Is there a "Bound" Entanglement in Nature?', Phys. Rev. Lett. 80, 5239.

Horodecki, M., Horodecki, P., and Horodecki, R. (2000a), 'Asymptotic Entanglement Manipulations Can Be Genuinely Irreversible', Phys. Rev. Lett. 84, 4260.

Horodecki, R., Horodecki, M., and Horodecki, P. (2000b), 'On Balance of Information in Bipartite Quantum Communication Systems: Entanglement-Energy Analogy', quant-ph/0002021.

Lo, H., Popescu, S., and Spiller, T. (1998), Introduction to Quantum Computation and Information, Singapore: World Scientific. 
Massar, S., Bacon, D., Cerf, N., and Cleve, R. (2000): 'Classical Simulation of Quantum Entanglement Without Local Hidden Variables', quant-ph/0009088. Maudlin, T. (1994), Quantum Non-Locality and Relativity, Blackwell: Oxford. Mermin, N. D. (1996), 'Hidden Quantum Non-Locality’, in R. Clifton (ed.) Perspectives on Quantum Reality, Kluwer Academic Publishers, pp. 57-71.

Nielsen, M. A. and Chuang, I. L. (2000), Quantum Computation and Quantum Information, Cambridge: Cambridge Univ. Press.

Peres, A. (1996), 'Collective Tests for Quantum Nonlocality', Phys. Rev. A, 54, 2685-2689.

Plenio, M. and Vedral, V. (1998), 'Teleportation, Entanglement and Thermodynamics in the Quantum World', Contemp. Phys. 39, 431-446.

Popescu, S. (1994), 'Bell's Inequalities versus Teleportation: What is Nonlocality?', Phys. Rev. Lett. 72, 797-799.

Popescu, S. (1995), 'Bell's Inequalities and Density Matrices: Revealing 'Hidden' Nonlocality,' Phys. Rev. Lett. 74, 2619-2622.

Popescu, S. and Rohrlich, D. (1992), 'Generic Quantum Non-Locality', Phys. Lett. A, 166, 293-297.

Popescu, S. and Rohrlich, D. (1997), 'Thermodynamics and the Measure of Entanglement', Phys. Rev. A 56, R3319-R3321.

Popescu, S. and Rohrlich, D. (1998), 'The Joy of Entanglement' in Lo, Popescu, and Spiller (eds.) (1998).

Preskill, J. (1998), Lecture Notes for Physics 229: Quantum Information and Computation, http://www.theory.caltech.edu/people/preskill/ph229/.

Schrödinger, E. (1935), 'Discussion of Probability Relations between Separated Systems', Proc. Camb. Phil. Soc. 31, 555-563. 
Schrödinger, E. (1936), 'Probability Relations between Separated Systems', Proc. Camb. Phil. Soc. 32, 446-452.

Shor, P., Smolin, J., and Terhal, B. (2000), 'Evidence for Nonadditivity of Bipartite Distillable Entanglement', quant-ph/0010054.

Steiner, M. (1999), 'Towards Quantifying Non-Local Information Transfer: FiniteBit Non-Locality', Phys. Lett. A 270, 239-244.

Vidal, G. and Cirac, J. I. (2001), 'Irreversibility in Asymptotic Manipulations of Entanglement', quant-ph/0102036.

Werner, R. (1989), 'Quantum States with Einstein-Podolsky-Rosen Correlations Admitting a Hidden-Variable Model,' Phys. Rev. A 40, 4277-4281.

Żukowski, M. (2000), 'Bell Theorem for Nonclassical Part of Quantum Teleportation', Phys. Rev. A, 62, 032101. 